\title{
Mathematical Estimation Methods and Models for Industrial Companies
}

\author{
Olga Stikhova* \\ Moscow State Technological University "STANKIN", RU-127055, Moscow, Russia
}

\begin{abstract}
The collateralized debt obligations and credit default swaps applications are shown in this paper. The industry obligations secondary market risk estimation methods are considered in this work. The new methods taking into account statistically significant parameters for industrial credit derivatives portfolio are offered for single-name investment risks numerical experiments realization. The mathematical estimation of tranche were shown. The single and multiple name default obligations necessary mathematical modeling methods and formulae for the industrial materials manufacturers derivative credit tools market are shown. It is determined that the portfolio of synthetic debt tools is made of the given parameters. The task of a loss derivative tranches mathematical estimation is solved. Late defaults raise the equity tranches payment required sums with high spreads, early defaults reduce. Also the functional characteristics required for an estimation huge debts problem solving are partly considered in this paper. The problem of the default modeling for market tools and numerical simulation of the obligations influence on conditions of current bistability mode are shown here. Some credit derivatives of industrial manufacturers are demonstrated in the modeling process of default as an example. It is found that the model is an additional factor help us to estimate the default opportunity.
\end{abstract}

\section{Inverse Distribution}

The present work is concerned with the problem of the default modeling for market tools and numerical simulation of the obligations influence on conditions of current bistability mode. By example some credit derivatives of industrial manufacturers are demonstrated in the modeling process of default [1]. One of the market basic products for hedging credit risk is credit default swap (CDS). CDS forms the basis for more complex structures. In any credit event the credit protection seller carries out a rate CDS payment value for the protection buyer benefit for the default emitter obligation determined in the agreement. Let $\left(X_{n}, n \in \square\right)$ stationary casual sequence given on some filtered stochastic space $\left(\Omega, \mathfrak{I},\left(\mathfrak{I}_{n}\right)_{n \geq 0}, P\right)$. Suppose that $X_{n}$ behavior dynamics is described as in [2]. Consider that the conditional average $\mu_{n}$ and the volatility $\sigma_{n}$ are $\mathfrak{I}_{n-1}$ measurable. Let's take $F_{X}(x)$ of $\left(X_{n}\right)$ as distribution process function and $F_{X_{n+1}+\ldots+X_{n+k} \mid \Im_{n}}(x)$ is distribution conditional function for subsequent $k$ measurements on a $\mathfrak{J}_{n}$ filtration. We find quantile of such distributions for where $\mathrm{N}$ can be large enough and enter also conditional mathematical expectation. To calculate them we should construct the appropriate models of dynamics

\subsection{The problem statement}

\subsubsection{Dynamic models}

The distribution conditional function quantile expression

$$
\begin{aligned}
& F_{X_{n+1}+\ldots+X_{n+k} \mid \Im_{n}}(x) \text { is } \\
& \quad x_{q}^{n}(k)=\inf \left(x \in \square: F_{X_{n+1}+\ldots+X_{n+k} \mid \Im_{n}}(x) \geq q\right) .
\end{aligned}
$$

Take also a conditional mathematical expectation

$$
S_{q}^{n}(k)=E\left(\sum_{j=1}^{k} X_{n+j} \mid \sum_{j=1}^{k} X_{n+j}>x_{q}^{n}(k), \mathfrak{I}_{n}\right)
$$

As

$$
F_{X_{n+1} \mid \mathfrak{I}_{n}}(x)=P\left(\sigma_{n+1} Z_{n+1}+\mu_{n+1} \leq x \mid \mathfrak{\Im}_{n}\right)=F_{Z}\left(\left(x-\mu_{n+1}\right) / \sigma_{n+1}\right),
$$

then an expression of $x_{q}^{n}$ and $S_{q}^{n}$ are

$$
\begin{gathered}
x_{q}^{n}=\mu_{n+1}+\sigma_{n+1} Z_{q}, \\
S_{q}^{n}=\mu_{n+1}+\sigma_{n+1} E\left(Z \mid Z>z_{q}\right),
\end{gathered}
$$

Corresponding author: olgitast2011@mail.ru 
where $Z_{q}$ - distribution function quantile $F_{Z}(z)$. To calculate (4) we should construct the appropriate dynamic models for $\mu_{n}$ and $\sigma_{n}$. We offer to use model $A R(1)[1]$ for conditional average $\mu_{n}$ and model $\operatorname{GARCH}(1,1)\left[2\right.$ ] for volatility $\sigma_{n}$

$$
\begin{gathered}
\mu_{n}=\phi X_{n-1}, \\
\sigma_{n}^{2}=\alpha_{0}+\alpha_{1} \varepsilon_{n-1}^{2}+\alpha_{2} \sigma_{n-1}^{2},
\end{gathered}
$$

where $\quad \varepsilon_{n}=X_{n}-\mu_{n}, \quad \alpha_{0}>0, \alpha_{1}>0, \alpha_{2}>0$, $\alpha_{2}+\alpha_{1}<1,|\phi|<1$.

The $\tilde{\theta}=\left(\tilde{\phi}, \tilde{\alpha}_{0}, \tilde{\alpha}_{1}, \tilde{\alpha}_{2}\right)$ parameters estimations can be received by a maximum likelihood method. The conditional average $\left(\tilde{\mu}_{1}, \ldots, \tilde{\mu}_{n}\right)$ and volatility $\left(\tilde{\sigma}_{1}, \ldots, \tilde{\sigma}_{n}\right)$ estimations can be calculated by consecutive substitution in expressions (5) at the appropriate choice of initial meanings. Then the excess meanings and conditional average estimations are

$$
\begin{gathered}
\left(z_{1}, \ldots, z_{n}\right)=\left(\frac{x_{1}-\tilde{\mu}_{1}}{\tilde{\sigma}_{1}}, \ldots, \frac{x_{n}-\tilde{\mu}_{n}}{\tilde{\sigma}_{n}}\right) \\
\tilde{\mu}_{n+1}=\tilde{\phi} x_{n}, \tilde{\sigma}_{n+1}^{2}=\tilde{\alpha}_{0}+\tilde{\alpha}_{1} \tilde{\varepsilon}_{n}^{2}+\tilde{\alpha}_{2} \tilde{\sigma}_{n}^{2},
\end{gathered}
$$

where $\tilde{\varepsilon}_{n}=x_{n}-\tilde{\mu}_{n}$.

The distribution function considerably differs from normal distribution, assumed in standard GARCH-model as the financial temporary series analysis has shown [3].

\subsubsection{Loss industry obligations secondary market}

The loss check of the industry obligations of the secondary market and the estimation methods are submitted in this paper, the concept "derivative" of standard financial actives is considered. The collateralized debt obligations (CDO) and credit default swaps (CDS) are appreciated, and also their estimation problem solving functional characteristics are considered.

Necessary modeling methods and mathematical formulae of the single and multiple name credit default swaps are shown for the market of the industrial materials manufacturers. It is determined that the portfolio of synthetic debt tools is made of the given parameters. The CDS spread is fixed so its meaning of a bonus part and the parts of protection were equal:

$$
S=\frac{E\left[\int\left(1-R_{t}\right) \lambda_{t} e^{-\int^{t}\left(r_{u}+\lambda_{u}\right) d u} d t\right]}{E\left[\sum_{i=1}^{M} e^{-\int_{i}^{T_{i}}\left(r_{t}+\lambda_{t}\right) d t} \Delta_{i}+\sum_{i=1}^{M} \int_{T_{i-1}}^{T_{i}}\left(t-T_{i-1}\right) \lambda_{t} e^{-\int^{t}\left(r_{u}+\lambda_{u}\right) d u} d t\right]}
$$

$$
\text { Default intensity: } \lambda=\frac{1}{\Delta} \ln \left(\frac{s \Delta}{(1-R)}+1\right)
$$

\subsection{Tranches estimation}

The single-name default mathematical model is

$$
\tau=\inf \left\{t: N_{t}=1\right\},
$$

where $\tau$-default time, $N=\left(N_{t}\right)_{t \geq 0}$ - default process, $\lambda=\left(\lambda_{t}\right)_{t \geq 0}$ - right non-negative continuous and left limited function of default intensity.

The default probability before the time $t$, in view of the default absence of the emitter and market information up to the moment of time s:

$$
P_{\text {def }}(t \mid s)=1-E\left[e^{-\int^{t} \lambda_{u} d u} \mid F_{s}\right]
$$

The single-name manufacturer tranches inverse distribution estimation methods are considered. The task of a loss derivative tranches mathematical estimation is solved [3]. Late defaults raise the equity tranches payment required sums with high spreads, early defaults reduce.

Spread CDO:

$$
s=\frac{\int_{0}^{T} E\left[e^{-\int^{S} r_{u} d u} d L_{s}^{\left(K_{1} \cdot K_{2}\right)}\right]}{\sum_{i=1}^{M} E\left[e^{-\int_{i}^{T_{i}} r_{u} d u} \Delta_{i} \frac{2-L_{T_{i-1}}^{\left(K_{1}, K_{2}\right)}-L_{T_{i}}^{\left(K_{1}, K_{2}\right)}}{2}\right.}
$$

expected losses:

$$
\begin{aligned}
& E L_{\left(K_{1}, K_{2}\right)}(t)=\frac{1}{K_{2}-K_{1}} \int_{K_{1}}^{1}\left(\min \left(x, K_{2}\right)-K_{1}\right) d F(t, x)= \\
& =\frac{1}{K_{2}-K_{1}}\left(\int_{K_{1}}^{1}\left(x-K_{1}\right) d F(t, x)-\int_{K_{2}}^{1}\left(x-K_{2}\right) d F(t, x)\right)
\end{aligned}
$$

distribution of losses :

$$
F_{\infty}(x)=P[X \leq x]=\Psi\left(\frac{\sqrt{(1-\rho)} \Psi^{-1}(x)-K}{\sqrt{\rho}}\right)
$$

\section{Modelling}

In the given work the new mathematical estimation formulae for inverse distribution of tranches are developed. The multiparametrical model based on generalized hyperbolic copula with generalized hyperbolic borders (CGH), taking into account the 
various mixed distribution and thus, various boundary areas distributions, keeping desirable one-dimensional distribution $\mathrm{GH}$ properties is offered.

The GH-copula density $f_{G H_{d}}(x, \lambda, \chi, \psi, \mu, \Sigma, \gamma)$ depends on some parameters such as $K_{\lambda}$ - the modified Bessel function of the third rank with an applicable domain range $\square$, heavy tail $\lambda$, $\chi$ - scaling rang, $\psi$-form, $\mu$-shift, $\Sigma$ - covariation matrix, $\gamma$ - skewness.

The parameters $\mu \in \square ; \chi \geq 0, \psi \geq 0$, if $\lambda<0$; $\chi>0, \psi>0$, if $\lambda=0 ; \chi \geq 0, \psi>0$, if $\lambda>0$ and $|\Sigma|:=1$ for recognition. The vector $X \square G H_{d}\left(\lambda, \chi, \psi, \mu, A A^{\prime}, \gamma\right)$ is defined as

$$
X \stackrel{d}{=} \mu+\omega \gamma+\sqrt{\omega} A Z
$$

$d$

where $Z=N_{k}\left(0, I_{k}\right), \quad \omega \square G I G(\lambda, \chi, \psi)$ - positive scalar casual variable, not dependent from matrixes $Z, A \in \square^{d \times k}$ and $\mu, \gamma$ are belong to $\square^{d}$.

We assume, that the indefinitely large portfolio with an active profit corresponds to copula model CGH, table 1.

Table 1. Various distributions tranches DJ iTraxx estimation.

\begin{tabular}{|c|c|c|c|c|c|}
\hline$\%$ & Market & Gauss & $\begin{array}{l}\mathrm{t}(4)-\mathrm{t}(4) \\
\mathrm{t}(3)-\mathrm{t}(3)\end{array}$ & $\begin{array}{l}\text { NIG1 } \\
\text { NIG2 }\end{array}$ & $\mathrm{CGH}$ \\
\hline $0-3$ & $24.3 \%$ & $24.3 \%$ & $24.3 \%$ & $\begin{array}{l}24.3 \\
\%\end{array}$ & $24.3 \%$ \\
\hline $3-6$ & 157.3bp & $\begin{array}{l}238.7 \\
\text { bp }\end{array}$ & $\begin{array}{l}\text { 167.3bp } \\
\text { 148bp }\end{array}$ & $\begin{array}{l}157.3 \\
\text { bp } \\
157.3 \\
\text { bp } \\
\end{array}$ & 157.3bp \\
\hline $6-9$ & 47.1bp & $\begin{array}{l}59.01 \\
\text { bp }\end{array}$ & $\begin{array}{l}\text { 37.21bp } \\
44.7 \mathrm{bp}\end{array}$ & $\begin{array}{l}37.6 b \\
p \\
37.7 b \\
p\end{array}$ & 48.1bp \\
\hline $9-12$ & 21.7bp & $\begin{array}{l}18.9 b \\
p\end{array}$ & $\begin{array}{l}\text { 28.98bp } \\
27.8 b p\end{array}$ & $\begin{array}{l}30.1 b \\
\text { p } \\
30.23 \\
\text { bp }\end{array}$ & 21.7bp \\
\hline $\begin{array}{l}12- \\
22\end{array}$ & 11.73bp & $\begin{array}{l}8.78 b \\
p\end{array}$ & $\begin{array}{l}\text { 16.66bp } \\
17.7 b p\end{array}$ & $\begin{array}{l}17.73 \\
\text { bp } \\
17.1 b \\
p\end{array}$ & 11.7bp \\
\hline $\begin{array}{l}\text { absol } \\
\text { ute } \\
\text { error }\end{array}$ & $24.3 \%$ & $\begin{array}{l}115,3 \\
\text { bp }\end{array}$ & $\begin{array}{l}\text { 31.85bp } \\
\text { 25.81bp }\end{array}$ & $\begin{array}{l}21.34 \\
\text { bp } \\
21.75 \\
\text { bp }\end{array}$ & 8.3bp \\
\hline $\begin{array}{l}\text { corre } \\
\text { latio } \\
n\end{array}$ & 157.3bp & $17.4 \%$ & $\begin{array}{l}32.71 \text { bp } \\
27.81 \text { bp }\end{array}$ & $\begin{array}{l}16.3 \\
\% \\
15.8 \\
\% \\
\end{array}$ & $23.9 \%$ \\
\hline
\end{tabular}

\subsection{Research process}

Take $\Omega(x)=K-v F_{G H}^{-1}(x)-m$ and contingent loss $Y=y$, part of the default papers in portfolio $X$ and $p(y)$ we have:

$$
\begin{aligned}
& \lim _{N \rightarrow \infty} P(X \geq x)=P(a(Y) Y \leq \Omega(x), Y \leq \theta)+ \\
& +P(a(Y) Y \leq \Omega(x), Y>\theta)
\end{aligned}
$$

And then the distribution integrated function is

$$
\begin{aligned}
& F_{\infty}(x)=1-\lim _{N \rightarrow \infty} P(X \geq x)= \\
& =1-\left[\begin{array}{l}
F_{G H}\left(\min \left(\frac{\Omega(x)}{\sqrt{a}}, \theta\right)\right)+ \\
+1_{\frac{\Omega(x)}{\sqrt{b}}>\theta}\left(F_{G H}\left(\frac{\Omega(x)}{\sqrt{b}}\right)-F_{G H}(\theta)\right)
\end{array}\right]
\end{aligned}
$$

During the research of the single-name quotation process it is marked that the distribution $\mathrm{F}(\mathrm{x})$ is not trivial and depends on the various emitters defaults in a base portfolio as the occurrence is disproportionate default losses results to heavy tails. In a case of CDO also it is necessary to take into account default time as the payments depend on cost which decreases during the contract period if default occurs [4].

It is offered the multiple names credit default probability model. In a case of $n$ emitters during the time $\tau^{i}$ for each emitter $i, i=1 \ldots, n$ the default indicator function $N_{t}^{i}=1_{\left\{\tau^{i} \leq t\right\}} \mathbf{i}=1, \ldots$, n. The default time is determined as the first moment of time, when the

$$
-\int^{t} \lambda_{s}^{i} d s
$$

function meaning $\left(e^{0}\right)_{t \geq 0}$ becomes less then $\mathrm{U}^{\mathrm{i}}$ uniform distributed at $[0,1]$ :

$$
\tau^{i}=\inf \left\{t: e^{-\int_{0}^{t} \lambda_{s}^{t} d s} \leq U^{i}\right\}
$$

where the $U=\left(U^{1}, \ldots, U^{n}\right)$ dependence fixed by copula $C$ multidimensional function with standard onedimensional margins.

The probability of non-defaults up to the moment of time $t$ at default absence of the emitters $k \neq j$ up to the moment of time $s$, default of the emitter $\mathrm{j}$ at the moment of time $u$ and the presence of the large number of information parameters is

$$
\tilde{P}_{\text {surv }}^{i,-j(u)}(t \mid s)=\frac{\frac{\partial}{\partial x_{j}} C\left(\gamma_{s}^{-i,-j}, \gamma_{t}^{i}, \gamma_{u}^{j}\right)}{\frac{\partial}{\partial x_{j}} C\left(\gamma_{s}^{-j}, \gamma_{u}^{j}\right)}
$$

\subsection{Default probability}


With the portfolio losses $x \in[0,1]$ and the default threshold value $K(t)=F_{G H}^{-1}(p(t))$, where $p(t)$ is neutral to risk default probability of each portfolio emitter

$$
p_{n}(y)=F_{G H_{i}}\left(\frac{K-a y}{\sqrt{1-a^{2}}}\right)
$$

the portfolio loss distribution is:

$$
F_{\infty}(t, x)=1-F_{G H_{d}}\left(\frac{1}{a}\left(K(t)-\sqrt{\left(1-a^{2}\right)} F_{G H_{i}}^{-1}(x)\right)\right)
$$

The tranche expected losses [5]:

$$
E L_{\left(K_{1}, K_{2}\right)}(t)=\frac{1}{K_{2}-K_{1}} \int_{K_{1}}^{K_{2}}\left(x-K_{1}\right) d F_{\infty}(t, x)+\left(1-F_{\infty}\left(t, K_{2}\right)\right)
$$

In this work single-name default probability model is used to the premium part estimation, the saved payments are taken into account, default time distribution is calibrated under the CDS rates.

For the basic portfolio CDO valuation the multiple-name default probability model is used [5]:

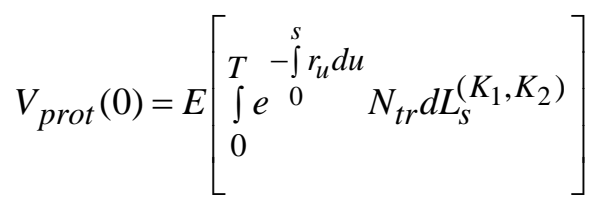

and

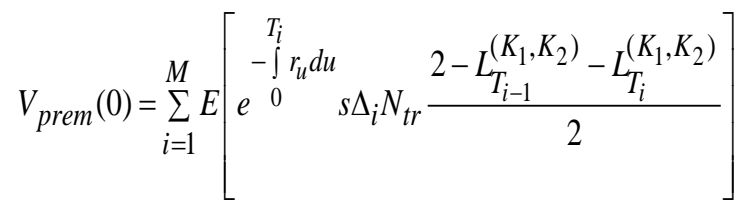

The value of protection is determined by the size of the expected tranche default losses and the value of tranche premium part is calculated as the current price of all expected payments on spread, where an interval in payments is $\Delta_{\mathrm{i}}=\mathrm{T}_{\mathrm{i}}-\mathrm{T}_{\mathrm{i}-1}$.

\subsection{Dependence illustrations}

To estimate the price of a base portfolio of CDS the single-name model of probability is used. The default time distribution was calibrated based on CDS. The credit default swap transaction is initiated at the zero moment of time with term of the expiration T., $\forall t \leq T$. All bonus payments are accomplished in a time interval $0<T_{1}<\ldots<T_{M}=T$ at the expected sum of payments $\mathrm{N}$ and annual spread rate $s$ and where $\lambda=\left(\lambda_{t}\right)_{t \geq 0}$ is a default intensity function of the appropriate emitter and discount is $E\left[e^{-\int^{T} r_{s} d s} \mid F_{t}\right]>0$.

In case of the default before the term of protection, the seller of protection should carry out compensatory payment $\left(1-R_{n}\right) N, \tau \leq T$.

At an estimation of a bonus part the saved payments are taken into account.

At the estimation of the CDO tranche price of a base portfolio the multiple -names default probability model is used [4].

There are no yet credit cases the CDO emitter regularly pays the premium (insurance payment) to the tranche investor.

In a default case the investor (the seller of protection) pays to the CDO emitter (buyer of protection) the rate of the suffered losses sum.

The following premium (insurance payment) is paid in view of a deduction of the sum of the losses.

The tranche CDO protection price covers the losses of a base portfolio between two given threshold meanings $\mathrm{K} 1$ and $\mathrm{K} 2$.

$N_{p}$ is an expected income of the appropriate portfolio, annual $\mathrm{s}$ tranche spread and $N_{t r}=\left(K_{2}-K_{1}\right) N_{p}$ is an expected tranche income. Dates of payments are $0<T_{1}<\ldots<T_{M}=T$,

where $T$ is a term of the obligations execution, t0 $<\mathrm{t} 1$ is a date of an estimation. The bonus payments are carried out in time tk for the period of payment from tk-1 up to tk.

The premium in time tk is paid at stipulated cost at the given moment of time. The emitter $\mathrm{i}, 1 \leq \mathrm{i} \leq \mathrm{n}$ in a portfolio has a default with intensity $\lambda \mathrm{i}$.

The proportional trance default loss $L_{t}^{\left(K_{1}, K_{2}\right)}$ up to the moment of time $t \%$ from tranche nominal).

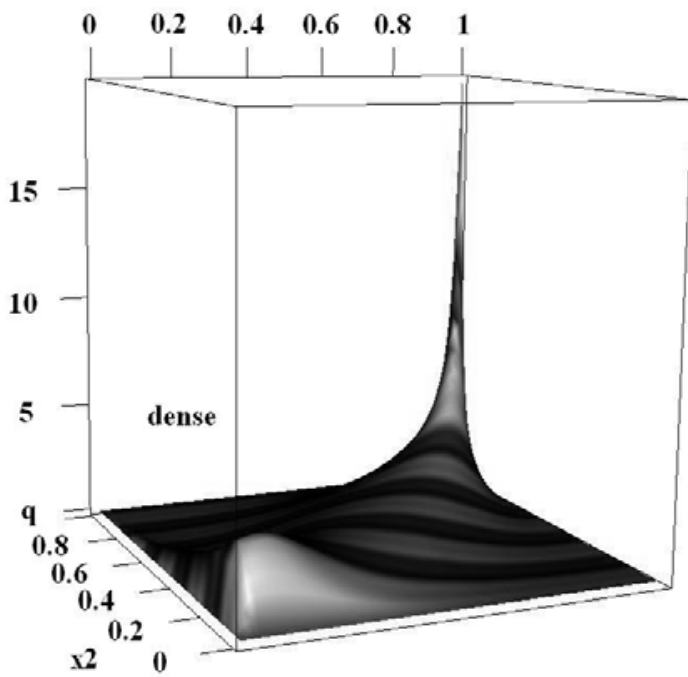

Fig. 1. GH-copula density $c_{G H}\left(u_{1}, \ldots, u_{d}\right)$. 
Thus cost parameters CGH model of credit derivatives depend on the limit independence and tail dependence better than used earlier multidimensional models (fig.3).

For GH-copula the density $f_{G H_{d}}\left(x, \lambda, \chi, \psi, \mu, \sum, \gamma\right)$ is:

$$
\begin{aligned}
& f_{G H_{d}}=\frac{(\sqrt{\psi \chi})^{-\lambda} \psi^{\lambda}\left(\psi+\gamma^{\prime} \Sigma^{-1} \gamma\right)^{\frac{d}{2}-\lambda}}{(2 \pi)^{\frac{d}{2}}\left|\sum\right| \frac{1}{2} K_{\lambda}(\sqrt{\psi \chi})} \times \\
& \times \frac{K_{\lambda-\frac{d}{2}}\left\{\sqrt{\left(\chi+(x-\mu)^{\prime} \Sigma^{-1}(x-\mu)\right)\left(\psi+\gamma^{\prime} \Sigma^{-1} \gamma\right)}\right\} e^{(x-\mu)^{\prime} \Sigma^{-1} \gamma}}{\left\{\sqrt{\left(\chi+(x-\mu)^{\prime} \Sigma^{-1}(x-\mu)\right)\left(\psi+\gamma^{\prime} \Sigma^{-1} \gamma\right)}\right\}^{\frac{d}{2}-\lambda}}
\end{aligned}
$$

where

$K_{\lambda} \quad$ - modified Bessel function of the third kind with the applicable domain $\square$,

$\lambda$ - tail index,

$\chi$ - scalability factor,

$\psi$ - form parameter,

$\mu$ - shift parameter,

$\Sigma$ - covariance matrix,

$\gamma$ - asymmetry parameter.

Parameters $\mu \in \square ; \chi \geq 0, \psi \geq 0$,

if $\lambda<0 ; \chi>0, \psi>0$, if $\lambda=0 ; \chi \geq 0, \psi>0$,

if $\lambda>0$ and $|\Sigma|:=1$ for an identification.

$\begin{array}{ccc}\text { Vector } & X \square G H_{d}\left(\lambda, \chi, \psi, \mu, A A^{\prime}, \gamma\right) \\ X=\mu+\omega \gamma+\sqrt{\omega} A Z, & \text { where } \quad \stackrel{d}{=} N_{k}\left(0, I_{k}\right),\end{array}$ $\omega \square G I G(\lambda, \chi, \psi) \quad$ - matrix $Z, A \in \square^{d \times k}$ independent positive scalar random variable and $\mu, \gamma$ are parameters at $\square^{d}$.

\section{Results}

The calculation methods of conditional quantile and the mathematical expectations of random stationary sequences for extreme values are considered in this work. For the description of the sequences characteristics dynamics we used parametrical models AR(1) and GARCH $(1,1)$. The generalized Pareto distribution is used as modeling function of conditional distribution. It is shown by experiments, that the offered approach is more effective, than the standard normal distribution [6].

The single and multiple-name default process mathematical modeling task for some industrial production manufacturers is solved. The correlation between the emitter credit derivative default probability in a portfolio and the tranche market cost. The credit derivatives market researches and analysis results are shown that the important empirical property is the correlation dynamics influence on a default time [7-9].

The computing experiments on parameters modeling of the default credit derivatives for industrial manufacturers are carried out both with the generated samples and with the real data. As opposed to earlier multiparameter models these methods and formulae allow to simulate simultaneously tail dependence and various boundary areas distributions. The results verification is done and they have shown high efficiency [10]. The one and several industrial credit derivative debt default process mathematical modeling task having the increase importance for the enterprises, corporations and other structures is solved.

The correlation between credit derivative default probability of the emitter in a portfolio and the grown-up tranche market cost growth fall are established at the equity tranche cost increase. The research results and the credit derivative market analysis have revealed the important empirical property of credit derivative market indices costs - influence of correlation dynamics on the default time.

Based on the developed models the new estimation algorithm of default credit derivative for industrial corporations is offered. It allows to make qualitative market researches of the credit derivatives. Given algorithm can be used on practice for one and several emitters.

\section{References}

1. A. Pagan, J. of Empirical Finance, 3(1), 15-102 (1996) https://doi.org/10.1016/0927-5398(95)00020-8

2. O.V. Stikhova, Vestnik MSTU «STANKIN». Scientific referenced journal, 4(23), 81 (2012) [in Russia] ISSN 2072-3172.

3. O.V. Stikhova, Fund. Phys. Math. Prob. T-T. Sys. Mod., 16, 97 (2014) [in Russia] ISBN 978-5-8037-0621-2.

4. R.F. Engle, Econometrica, 50(4), 987-1007 (1982) https://doi.org/10.2307/1912773

5. O.V. Stikhova, Mnps-2015, 46 (2015) [in Russia] ISBN-978-5-8037-0655-7.

6. D.M. Mason, The Heritage Foundation, 2262 (2009)

7. J. Sidenius, V. Piterbarg, L. Andersen, International Journal of Theoretical and Applied Finance, 11, 163-197 (2008)

8. A. Kalemanova, R. Werner, A Short Note on the Efficient Implementation of the Normal Inverse Gaussian Distribution (Risklab Germany. Nymphenburger Str., 2006)

9. A.J. McNeil, R. Frey, P. Embrechts, Quantitative Risk Management: Concepts, Techniques and Tools (Princeton University Press, 2005)

10. O.V. Stikhova, Fund. Phys. Math. Prob. T-T. Sys. Mod., 18(120) (2017) [in Russia] ISBN 978-5-8037-0710-3. 